\title{
Murine Colitis is Mediated by Vimentin
}

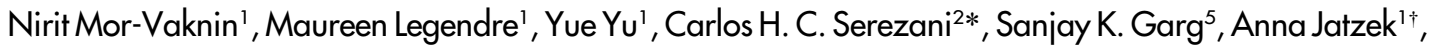 \\ Michael D. Swanson ${ }^{1,4}$, Marta J. Gonzalez-Hernandez', Seagal Teitz-Tennenbaum', \\ Antonello Punturieri ${ }^{2,65}$, N. Cary Engleberg ${ }^{1}$, Ruma Banerjee ${ }^{5}$, Marc Peters-Golden ${ }^{2,4}$, John Y. $\mathrm{Kao}^{7}$ \\ \& David M. Markovitz ${ }^{1,3,4}$
}

SUBJECT AREAS:

AUTOIMMUNITY

ACUTE INFLAMMATION

INTERMEDIATE FILAMENTS

BACTERIAL HOST RESPONSE

Received

12 October 2012

Accepted

10 December 2012

Published

9 January 2013

Correspondence and requests for materials should be addressed to

N.M.-V. (morkvak@ umich.edu)

* Current address: 650 Walnut street, research building 2 room 380, Indianapolis,

IN 46022.

$\uparrow$ Current address:

2015 Linden Drive,

Madison, WI 53706.

† Current address:

Campus Box 7295, Lineberger

Comprehensive Cancer

Center, University of

North Carolina, Chapel

Hill, NC 27599.

$\S$ Current address:

Two Rockledge Center

Suite 10018, 6071

Rockledge Drive MSC

7952, National Heart,

Lung and Blood Institute,

Bethesda, MD $20892-$

7952.
'Department of Internal Medicine, Division of Infectious Diseases, 5220 MSRB III, 1150 West Medical Center Drive, University of Michigan, Ann Arbor, MI 48109-5640, ²Division of Pulmonary and Critical Care, 6301 MSRB III, 1150 West Medical Center Drive, University of Michigan, Ann Arbor, MI 48109-5642, ${ }^{3}$ Cellular \& Molecular Biology Program, 2966 Taubman Medical Library, 1135 East Catherine Street, University of Michigan, Ann Arbor, MI 48109-5619, ${ }^{4}$ The Program in Immunology 5641 MS I, 1150 West Medical Center Drive, University of Michigan, Ann Arbor, MI 48109-5620, ${ }^{5}$ Department of Biological Chemistry, 3200 MSRB 3, 1150 West Medical Center Drive, University of Michigan, Ann Arbor, Ml 48109. 5606, ${ }^{6}$ The Research Section, Veterans Administration Medical Center, Ann Arbor, Michigan 48105-2303, ${ }^{7}$ Division of Gastroenterology, Department of Internal Medicine, University of Michigan, 6520A Medical Science Research Building 1, Ann Arbor, Ml 48109-5682.

Vimentin, an abundant intermediate filament protein, presumably has an important role in stabilizing intracellular architecture, but its function is otherwise poorly understood. In a vimentin knockout (Vim KO) mouse model, we note that Vim KO mice challenged with intraperitoneal Escherichia coli control bacterial infection better than do wild-type (WT) mice. In vitro, Vim KO phagocytes show significantly increased capacity to mediate bacterial killing by abundant production of reactive oxygen species (ROS) and nitric oxides, likely due to interactions with the p47phox active subunit of NADPH oxidase. In acute colitis induced by dextran sodium sulfate (DSS), Vim KO mice develop significantly less gut inflammation than do WT mice. Further, Vim KO mice have markedly decreased bacterial extravasation in the setting of DSS-induced acute colitis, consistent with decreased intestinal disease. Our results suggest that vimentin impedes bacterial killing and production of ROS, thereby contributing to the pathogenesis of acute colitis.

r he eukaryotic cytoskeleton is comprised of microfilaments, intermediate filaments, and microtubules ${ }^{1}$. Intermediate filaments are classified into three major groups: keratins, neurofilament-like proteins, and vimentin. Vimentin is the most abundant intermediate filament protein and is considered to play an important role in stabilizing intracellular architecture ${ }^{2}$. It is highly expressed in cells of mesenchymal origin, as well as in most transformed cell lines and tumors ${ }^{1,3}$. However, Vim KO animal models exhibit no overt phenotype at baseline, leading vimentin to be labeled as "the conundrum of the intermediate filament protein family". However, characterization of Vim KO mice under stress conditions, such as injury, has identified mild functional abnormalities ${ }^{4,5}$. Recent studies showing a defect in lymphocyte adhesion to endothelial cells in Vim KO mice ${ }^{6}$, further suggested that vimentin has specialized functions in different cell types.

The aim of this study was to define the role of vimentin in the innate immune response to bacterial pathogens. To this end, we examined the capacity of murine macrophages derived from Vim KO versus WT mice to generate ROS and to mediate bacterial killing. Our results show that lack of vimentin enhances ROS production and augments bacterial killing. Enhanced ROS production by Vim KO mice is correlated with a highly oxidizing environment, as measured by a reduced glutathione and oxidized glutathione ratio (GSH:GSSG) as an indicator of the cell redox potential. Our results suggest that vimentin suppresses ROS production by interaction with the p47phox active subunit of the prototypical phagocytic NADPH oxidase ${ }^{7-9}$.

Finally, we tested the functional relevance of these in vitro findings in two different murine models of acute colitis induced by E. coli or DSS. Consistent with our in vitro observations, lack of vimentin enhanced bacterial clearance and prolonged survival of mice in both models. Production of ROS by activated macrophages is essential to their capacity to mediate bacterial killing and thus constitutes an important part of the host defense system against invading micro-organisms. Taken together, these findings support a new concept whereby vimentin attenuates the production of ROS by activated macrophages, reducing their bacteriocidal capacity and supporting inflammation. 


\section{Results}

Role of vimentin in in vivo and in vitro bacterial killing. We have previously reported that activated human macrophages secrete vimentin into the extracellular space ${ }^{10}$. This secretion is regulated via inflammatory signaling pathways, with enhancement in response to tumor necrosis factor $\alpha(\mathrm{TNF} \alpha)$ and reduction by interleukin-10 (IL-10). Furthermore, anti-vimentin antibodies have been found to reduce the capacity of activated human macrophages to produce oxidative metabolites and to mediate bacterial killing, suggesting that vimentin plays a role in modulation of the innate immune response $\mathrm{e}^{10}$.

To test this hypothesis, we employed an established animal model of $E$. coli septicemia ${ }^{11}$ to determine whether lack of vimentin changes the capacity of the mice to mediate in vivo bacterial killing. Mice were challenged with a lethal dose of the E. coli strain J96 via intraperitoneal injection. Contrary to expectation, Vim KO mice demonstrated significantly lower levels of $E$. coli in the blood compared with WT mice (Figure 1A). These findings were associated with reduced mortality in Vim KO mice as compared to WT controls (Figure 1B, p < 0.05), further suggesting that lack of vimentin confers resistance to bacterial septicemia in a murine model. The difference in the results between the study employing anti-vimentin antibodies (9) and the genetic studies shown here may be due to inability of the antibodies to fully neutralize extracellular vimentin, or the elimination of both extracellular and intracellular vimentin using vimentin $\mathrm{KO}$ mice.

To investigate the potential mechanisms underlying enhanced bacterial clearance in Vim KO mice, we examined the capacity of peritoneal macrophages (PM) derived from Vim KO versus WT mice to mediate in vitro phagocytosis and killing of E. coli. Bacterial phagocytosis assays (Figure 1C) demonstrated no significant differences between the two groups. However, bacterial killing assays revealed that the macrophages of Vim KO mice exhibited significantly improved E. coli killing as compared to WT macrophages (Figure 1D, p < 0.05), indicating that lack of vimentin augments the ability of PM to kill E. coli in vitro.

Macrophages lacking vimentin produce more superoxide and nitric oxide. Reactive oxygen species (ROS) are important mediators of bacterial killing and serve as the first line of defense against bacterial invasion ${ }^{12,13}$. Previous work in other systems has suggested that vimentin modulate ROS production ${ }^{14,15}$. To test whether lack of vimentin promotes bacterial killing in macrophages via regulation of ROS production, we evaluated ROS levels in macrophages derived from Vim $\mathrm{KO}$ versus WT mice. PM were tested for production of nitric oxide (NO) in response to stimulation with LPS and IFN- $\gamma$, demonstrating that macrophages derived from Vim KO mice released higher levels of NO as compared with WT controls (Figure 2A, p $<0.005,>6$ fold difference). Further, PMA stimulation of peritoneal cells obtained from Vim $\mathrm{KO}$ mice produced higher amounts of superoxide $\left(\mathrm{O}_{2}{ }^{-}\right.$and $\left.\mathrm{H}_{2} \mathrm{O}_{2}\right)$ as compared to WT controls (Figure $2 \mathrm{~B}, \mathrm{p}<0.05,>5$ fold difference). No difference in superoxide dismutase levels was detected between Vim KO and WT macrophages (data not shown). Lack of vimentin also enhanced the capacity of bone marrow macrophages (BMM) to generate $\mathrm{H}_{2} \mathrm{O}_{2}$, with increased levels of this ROS being detected in the supernatants of Vim KO versus WT cells (Figure 2C). These findings were observed using unstimulated as well as PMA-stimulated BMM and were seen irrespective of the PMA concentration used for stimulation. Thus, macrophages that lack vimentin produce significantly more ROS than do cells derived from WT mice.
A

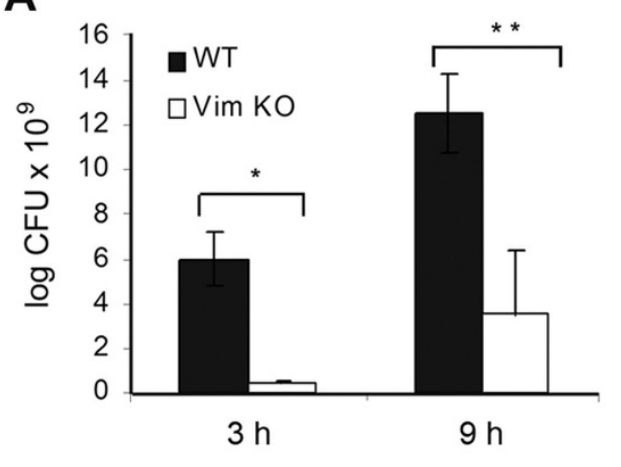

C

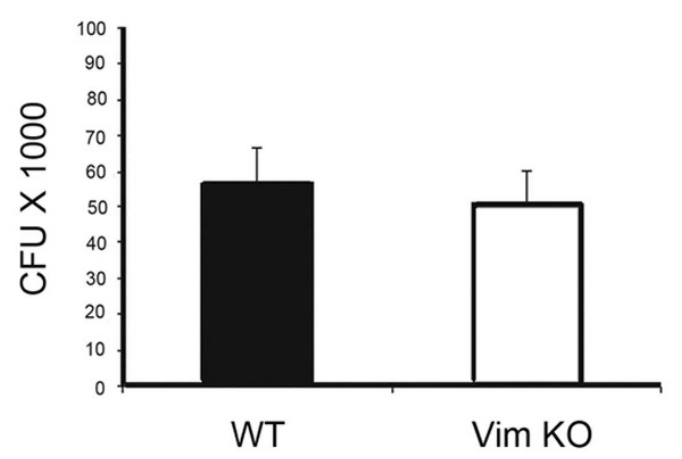

B
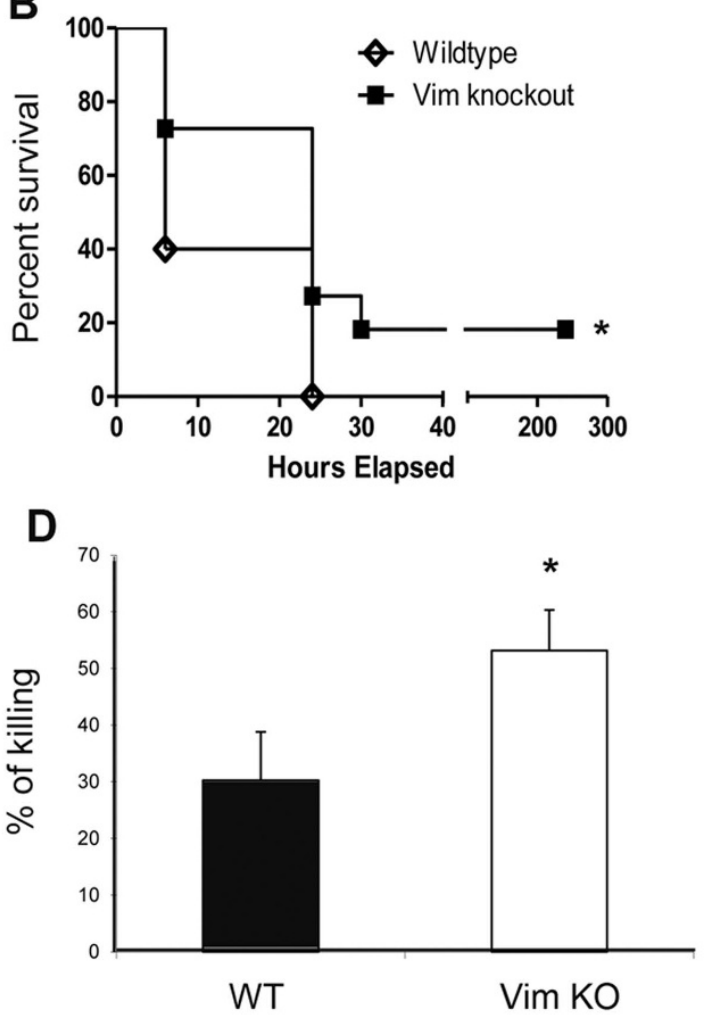

Figure 1 Vimentin inhibits the killing of $\boldsymbol{E}$. coli. (A). Vim KO $(\mathrm{n}=7)$ and WT mice $(\mathrm{n}=4)$ were injected IP with a lethal dose of $E$. coli. Blood samples collected 3 and 9 hours after injection were cultured for CFU (bacterial counts) $\left({ }^{*} \mathrm{P}<0.05\right.$ and ${ }^{* *} \mathrm{P}<0.05$, respectively). Data are representative of two independent experiments. (B). Survival curves generated using the log-rank test represent cumulative results obtained in two independent experiments (total Vim KO mice $\mathrm{n}=11$, total WT mice $\mathrm{n}=10$ ). ${ }^{*} \mathrm{P}<0.05$. (C, D). PM derived from Vim KO and WT mice were challenged with E. coli. Bacterial phagocytosis (C) and killing assays (D) were conducted as detailed in the Material and Methods section. * P $<0.05$. Data represent the average of two independent experiments \pm SEM of samples derived from a total of 7 mice. 

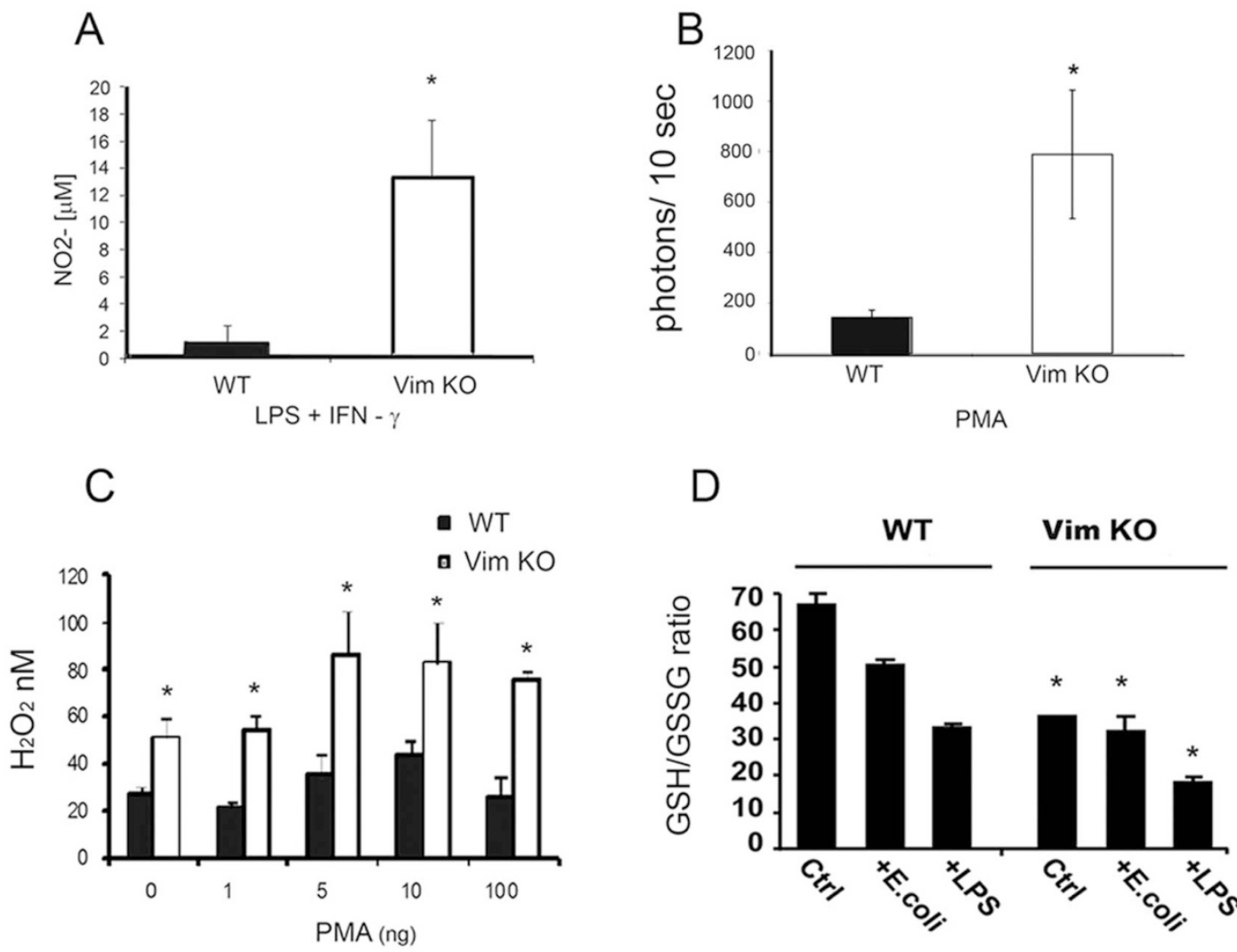

Figure $2 \mid$ Lack of vimentin increases production of ROS by murine macrophages. (A) PM derived from Vim KO $(n=7)$ or WT mice $(n=6)$ were stimulated with LPS and IFN- $\gamma$. Cell culture supernatants were collected and analyzed for nitric oxide. $* \mathrm{P}<0.005$. Data shown represent the average of 3 independent experiments. (B) PM derived from Vim KO and WT mice $(\mathrm{n}=8)$ were stimulated with PMA in the presence of luminol. Superoxide production was quantified by measuring light emission at the indicated time points after stimulation. $* \mathrm{P}<0.05$. Data represent the average of 3 independent experiments. (C). BMM derived from Vim KO and WT mice were incubated with or without increasing concentrations of PMA prior to determining $\mathrm{H}_{2} \mathrm{O}_{2}$ concentration in the supernatants. Data represent 2 independent experiments performed in triplicate. ${ }^{*} \mathrm{P}<0.05$ for Vim $\mathrm{KO}$ versus WT. (D). BMM derived from Vim KO or WT mice were incubated in the presence or absence of E. coli or LPS for $12 \mathrm{~h}$ and then intracellular concentrations of GSH and GSSG were measured via HPLC. Data are representative of three independent experiments. ${ }^{*} \mathrm{P}<0.05$, comparing Vim KO to WT mice.

Glutathione plays an essential role in the modulation of the cellular response to redox changes due to ROS production levels. Reduction in the intracellular ratio of reduced versus oxidized glutathione (GSH:GSSG) is indicative of oxidative stress. For that reason, we measured the intracellular redox capacity of unstimulated macrophages versus macrophages stimulated by $E$. coli or LPS derived from the bone marrow of Vim KO or WT mice. In all three test conditions, Vim KO BMM had significantly lower GSH:GSSG ratios compared to WT macrophages (Figure 2D, $\mathrm{P} \leq 0.05)$.

To extend these findings to human cells, we used the fluorescent probe DCFH-DA to assess ROS production in vimentin knockdown (Vim KD) cells derived from the human monocytic cell line THP-1 (Figure 3A). Both fluorescent microscopy (Figure 3B) and flow cytometry analysis (Figure $3 \mathrm{C}$ ) demonstrated that Vim KD cells produced more ROS than did control cells. In support of these findings, the levels of NADPH, a reducing agent that is produced by cells in response to oxidative stress and provides protection from ROS toxicity, were significantly higher in Vim KD cells compared to WT cells or cells transduced with a control shRNA vector (Figure 3D). Together, these results indicate that lack of vimentin enhances production of ROS by both mouse and human macrophages. Since ROS are critical for effective bacterial clearance, this finding likely accounts for the superior capacity of Vim KO macrophages to kill bacteria.
Vimentin interacts with p47phox. NADPH oxidase is the major enzyme responsible for generating $\mathrm{ROS}^{7-9}$. It catalyzes the oneelectron reduction of oxygen to superoxide $\left(\mathrm{O}_{2}{ }^{-}\right)$, which is the precursor of a variety of other ROS. NADPH oxidase is a multicomponent enzyme comprised of flavocytochrome b558 (p22phox and gp91phox), p47phox, p67phox, p40phox, and the small GTPase Rac 1/2. Upon activation of phagocytic cells, the cytosolic p47phox - p67phox - p40phox complex translocate to the membrane where it associates with the flavocytochrome b558 and assembles the active NADPH oxidase complex. In particular, the p47phox subunit is crucial to the in vivo assembly and function of the NADPH oxidase complex as genetic defects in p47phox are known to cause chronic granulomatous disease due to inefficient clearing of certain bacteria and fungi ${ }^{16,17}$. Previous studies, using polymorphonuclear leukocytes and endothelial cells, have suggested that the cytoskeleton may be involved in regulation of the translocation of the p47phox - p67phox - p40phox complex to the plasma membrane ${ }^{8}$. Therefore, we investigated whether vimentin interacts with the p47phox subunit in the membrane of PMA-activated macrophages. To this end, we conducted coimmunopreciptation assays in the membranous fractions of WT BMM before and after PMA-stimulation. Immunoblotting analysis of the vimentin pull-down precipitates in PMA-stimulated cells detected p47phox (Figure 4A). P47phox was not detected when an anti-IgG control antibody was used for immunoprecipitation (left 
A

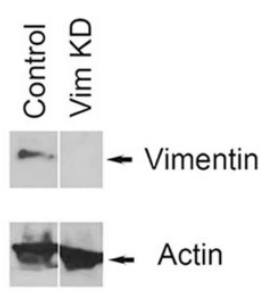

B

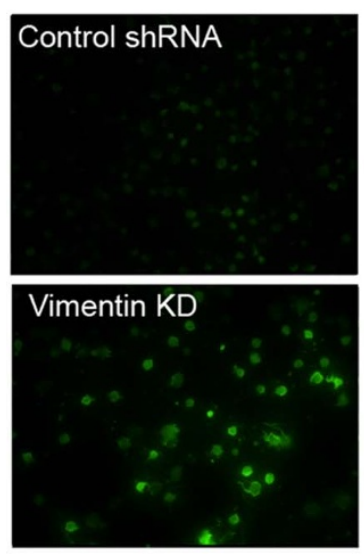

PMA
C

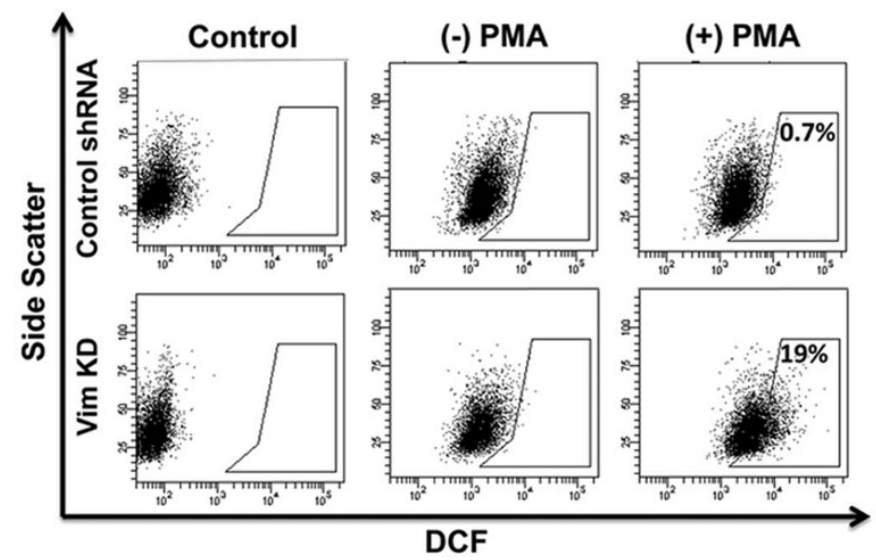

D

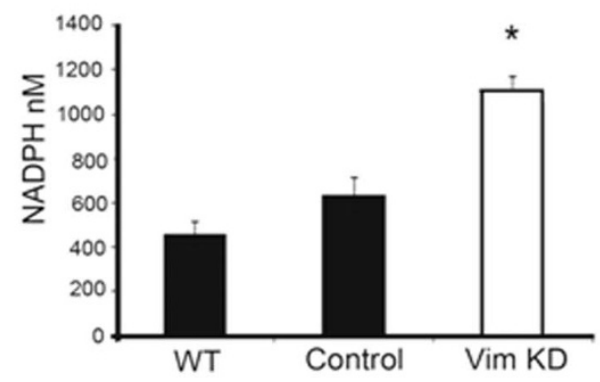

Figure 3 Knocking down vimentin expression in human macrophages increases production of ROS. (A) Western blot confirming knockdown of vimentin expression in the human monocytic cell line THP-1 transduced with shRNA targeting vimentin. (B). Fluorescence microscopy demonstrating levels of ROS detected by DCFH-DA in PMA-stimulated THP-1 cells transduced with an empty lentiviral vector (Control shRNA) or an shRNA targeting vimentin (Vim KD). (C). Flow cytometry analysis of the cells described in B. Data represents 2 independent experiments. (D). NADPH levels were measured in the WT human monocytic cell line THP-1, or THP-1 cells transduced with either an empty lentiviral vector (Control) or a vector expressing an shRNA targeting vimentin (Vim KD) after overnight serum deprivation. Data represent 2 independent experiments preformed in triplicate. ${ }^{*} \mathrm{P}<0.05$ comparing Vim KD cells to the control or wild-type cells.

lane) or when immunopreciptation was performed in macrophages derived from Vim $\mathrm{KO}$ mice (Figure 4A, right panel). Input analysis (Figure 4B) confirmed that $\mathrm{p} 47$ phox was present in the membranous fractions of activated Vim KO cells. In contrast to the membranous fraction, vimentin-p47phox interaction was not detected by immunoprecipitation experiments in the cytosolic fractions of the cells (data not shown). Moreover, confocal immunofluorescence microscopy analysis demonstrated co-localization of p47phox and vimentin at the plasma membrane of WT BMM (Figure 4C). This colocalization was detected primarily after PMA stimulation of the cells. Thus, it appears that vimentin and p47phox interact at the plasma membrane of activated macrophages. We postulate that this interaction interferes with the assembly process or with the functional activation of the NADPH oxidase complex. Further studies are needed in order to validate this hypothesis.

Mice lacking vimentin are protected from DSS-induced acute colitis. Defects in bacterial clearance have been linked to the pathophysiology of $\mathrm{IBD}^{18}$. In light of the information presented above, we explored whether decreased vimentin levels confer protection against IBD. Conversely, it has also been suggested, that increased ROS may stimulate gut inflammation and the development of $\mathrm{IBD}^{19}$, which would imply that loss of vimentin could exacerbate IBD. To address this question, we used the most common model of chemically-induced intestinal inflammation, namely, DSS-induced colitis. WT mice subjected to DSS treatment exhibited substantial weight loss (Figure 5A) associated with blood in the stool (Figure 5B) and had a high rate of mortality (Figure 5C). Histologic evaluation of colon sections harvested from WT mice showed severe ulceration of the gut mucosal lining and dense infiltrates of acute inflammatory cells, as shown in representative sections (Figure 5E). These findings were histologically quantified based on the leukocyte infiltration, crypt distortion, edema, and hemorrhage and presented as the Colitis Index (Figure 5D). In contrast, Vim KO mice that were exposed to the same treatment experienced only mild and transient weight loss, minor levels of blood in the stool, and no mortality. The mean Colitis Index of the colonic sections from Vim KO mice was significantly lower $(23.33 \pm 6.90)$ than that in WT mice $(38.00 \pm 2.00, \mathrm{p}<$ $0.05)$. These results show that lack of vimentin confers substantial resistance to chemically-induced acute colitis in mice. Interestingly, following DSS treatment, the levels of p47phox in protein extracts of colons from vim KO mice were much higher than those seen in WT mice. In contrast, levels of p67phox and p40phox were similar in control and DSS treated colons of both WT and Vim KO mice (Figure 5F). These results are consistent with the data presented above and further suggest that vimentin regulates the levels of p47phox in the inflamed colon. These results are surprising as previous studies suggested no role for $\mathrm{p} 47 \mathrm{phox}$ in DSS-induced colitis ${ }^{13}$. 
P47 phox
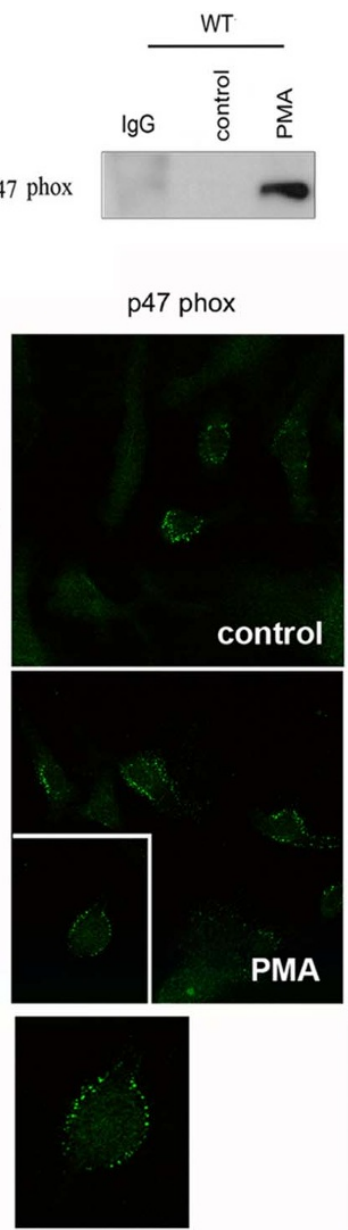

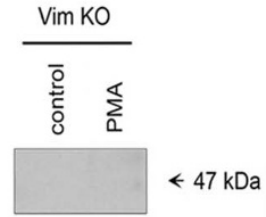

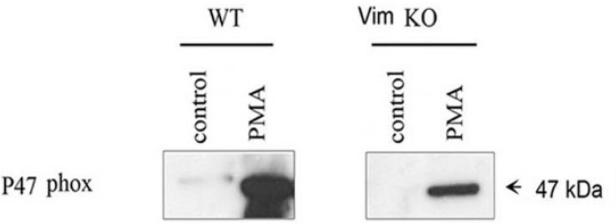

C
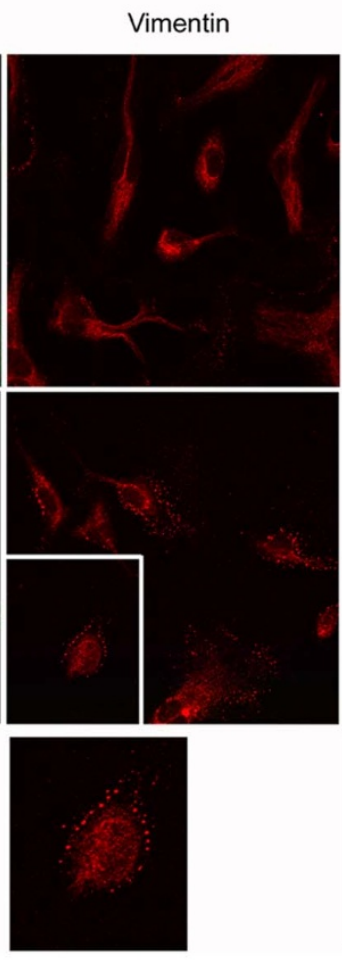

DAPI
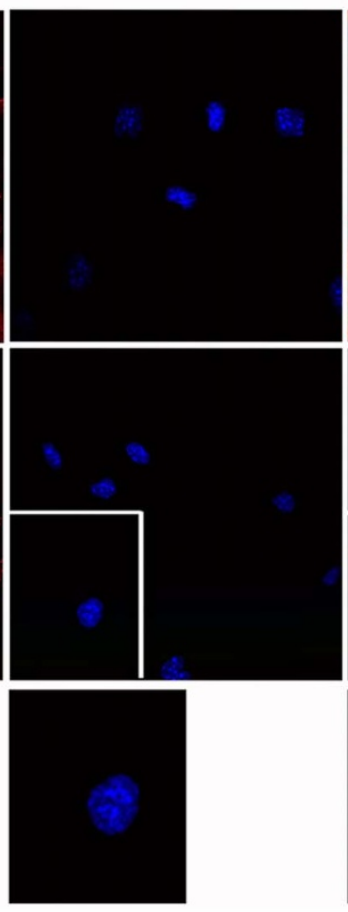

Merge
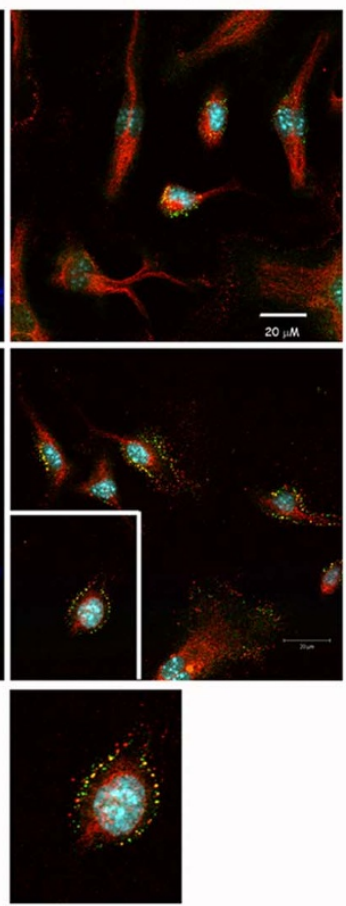

Figure $4 \mid$ p47phox co-immunoprecipitates and co-localizes with vimentin in the plasma membrane. (A). Vimentin was immunoprecipitated in the membranous fraction of unstimulated or PMA-stimulated BMM derived from WT mice using polyclonal anti-vimentin antibodies or a control IgG antibody. Immunoprecipitated proteins were analyzed by immunoblotting using a monoclonal anti-p47phox antibody. Immunopercipitation assays performed in the membranous fraction of BMM derived from Vim KO mice were used as a specificity control. (B). Immunoblotting for p47phox in the input samples described in A. Data are representative of 3 or more independent experiments. (C). Untreated (upper panel) and PMA-stimulated (lower panel) WT BMM were fixed and stained with antibodies targeting p47phox (green), vimentin (red) and DAPI (blue). Confocal microscopy analysis of the cells is shown at a X 63 magnification.

DSS is known to be toxic to the epithelial cells in the basal crypts of the colonic mucosa and is thought to mediate inflammation by inducing tissue damage that allows bacterial infiltration originating from the gut lumen ${ }^{20}$. Consistent with this model, analysis of bacterial loads in cultures derived from mesenteric lymph nodes demonstrated a significantly reduced level of bacterial infiltration in Vim $\mathrm{KO}$ as compared with WT mice (Figure 5G). These results show that Vim KO mice have a greater capacity to prevent bacterial infiltration compared with WT mice and are consistent with the in vivo and in vitro bacterial killing results shown above (Figure 1). These findings also suggest that enhanced bacterial killing protects Vim KO mice from DSS-induced colitis, at least in part.

\section{Discussion}

As vimentin is a highly-expressed intermediate filament protein, it was surprising to observe no distinct phenotype in Vim KO mice when they were originally developed ${ }^{21}$. In subsequent years, several groups have demonstrated that the knockout mice do indeed have subtle phenotypes ${ }^{4,6}$. Functional abnormalities in Vim KO mice have surfaced upon exposure to stress conditions, such as injury. Glial fibrillary acidic proteins in astrocytes derived from Vim KO mice fail to assemble into a filamentous network ${ }^{22}$. Fibroblasts of Vim KO mice display impaired mechanical stability, migration, and contractile capacity in tissue culture, leading to impaired wound healing 4 . Further, vimentin has been implicated in modulation of the vascular tone and in facilitating lymphocyte adhesion and transcellular migration $^{23}$. Studies by Zou and Chi et $a l^{24,25}$, using human brain microvascular endothelial cells, identified vimentin as a receptor for IbeA+-mediated E. coli K1-signaling and invasion. This strain of $E$. coli is responsible for inducing bacterial meningitis in newborns. Vimentin did not affect the phagocytic capacity of macrophages in our study, possibly due to lack of IbeA expression in the J96 strain. The on-going mystery of vimentin physiological function and our previous observation that vimentin may be involved in modulating bacterial killing led to the experiments presented in this study. Here, we demonstrated that vimentin greatly augments killing of $E$. coli both in vitro and in vivo. We showed that vimentin has a strong suppressive effect on ROS, in both mouse and human macrophages, that likely account, at least in part, for the fact that lack of vimentin leads to enhanced bacterial killing. These results are consistent with previous studies showing that vimentin can modulate production of ROS using immortalized primary embryo fibroblasts derived from 
A

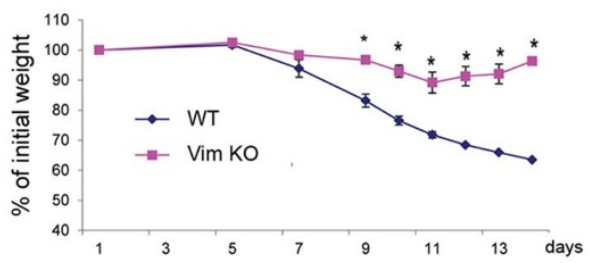

c

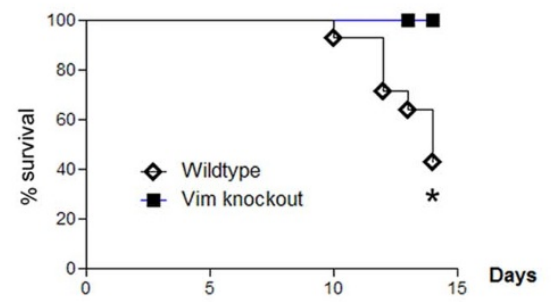

$\mathrm{F}$
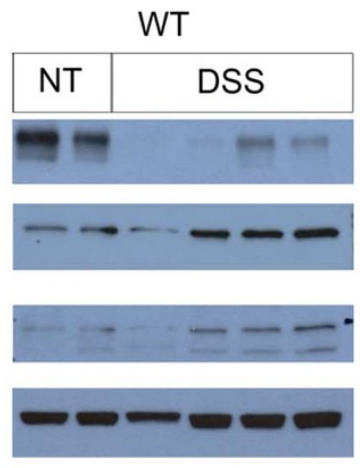

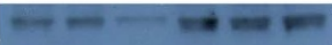

B

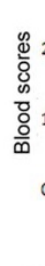

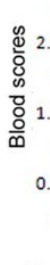

D

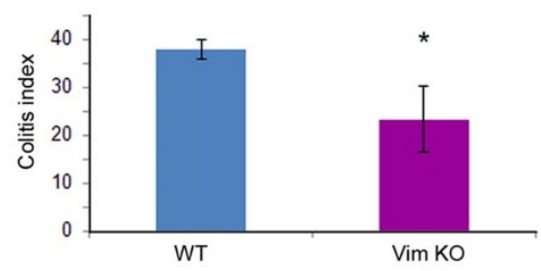

Vim KO

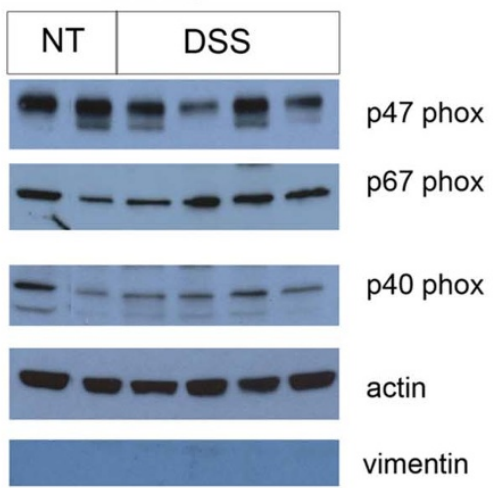

E
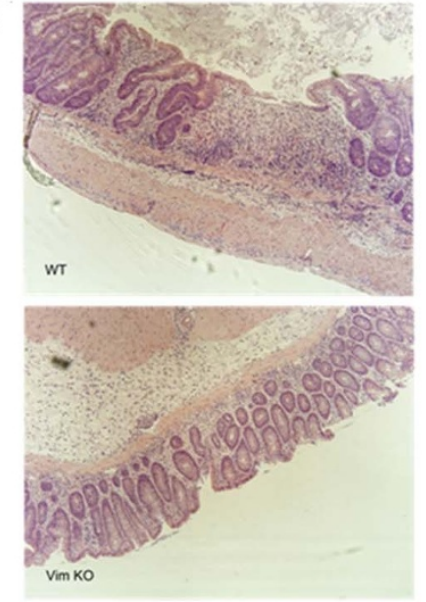

G

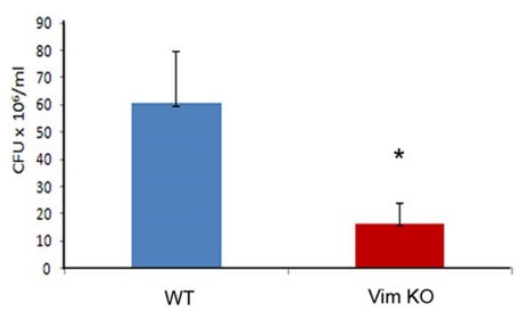

Figure 5 Vimentin mediates DSS-induced colitis. Vim KO and WT mice were treated with $4.5 \%$ DSS provided in the drinking water for 8 consecutive days. Mice were monitored for body weight $\left(\mathrm{A}, \mathrm{n}=3,{ }^{*} \mathrm{P}<0.005\right)$, presence of blood in the stools during the 14 days of treatment $\left(\mathrm{B}, \mathrm{n}=3,{ }^{*} \mathrm{P}<0.005\right)$, and survival $(\mathrm{C}, \operatorname{Vim} \mathrm{KO}(\mathrm{n}=20)$, WT $(\mathrm{n}=15) * \mathrm{P}<0.005)$. (D). Mice were sacrificed at day 14 and histological scores of colon sections were determined based on the colitis index $(45)\left(\mathrm{n}=3,{ }^{*} \mathrm{P}<0.05\right)$. Representative H\&E stained sections are shown in E. Data shown is representative of at least 3 independent experiments. (F). p47phox, p67phox and p40phox levels in extracts from the colons of untreated and DSS treated WT or Vim KO mice were analyzed by immunobloting using specific monoclonal antibodies. $(\mathrm{G})$. Bacterial counts of cultures derived from mesenteric lymph nodes (Vim KO $(\mathrm{n}=10)$, WT $\left.(\mathrm{n}=9) ;{ }^{*} \mathrm{P}<0.05\right)$, data represent the average of 2 independent experiments.

Vim KO mice, in which lack of vimentin leads to augmented production of ROS, resulting in oxidative damage ${ }^{26}$.

Bacterial infiltration derived from the gut is thought to be an important pathogenic mechanism in $\mathrm{IBD}^{18}$. Reduced bacterial killing and extravasation of bacteria from the lumen of the gut are also associated with the development of DSS-induced acute colitis in mice $^{18}$. In this study, we found that Vim KO mice are significantly less susceptible to DSS-induced colitis. These results are consistent with our data that shows increased production of ROS and increased capacity for bacterial killing in Vim KO mice. P47phox is known to be essential to the production of ROS by NADPH oxidase $\mathrm{o}^{7-9}$. Moreover, genetic defects in $\mathrm{p} 47 \mathrm{phox}$ has been established as a major cause of chronic granulomatous disease, in which ineffective bacterial killing may lead to severe unresolved infections ${ }^{16,17}$. In the report by Krieglstein et al. ${ }^{13}$, p47phox knockout mice respond to DSS treatment in a manner similar to wild type mice. This discrepancy may be due to the induction of colitis with a lower percentage of DSS, thereby reducing mortality rates in the strain of mice used by Kreiglstein et al. Interestingly, two different gene array analyses have shown increased vimentin expression in IBD, particularly in patients with ulcerative colitis (NCBI database, Gene Expression Omnibus GDS559/221729 and 201426). In addition, a recent study showed a significant increase in vimentin expression in patients with ulcerative colitis and Crohn's disease and further identified an interaction between NOD-2 and vimentin ${ }^{27}$. Consistent with these reports, our study identifies vimentin as a key mediator in the innate immune response to colonic bacteria and an important element in the pathogenesis of colitis. Further, our data suggest that vimentin may ultimately emerge as a therapeutic target for treatment of acute colitis phases associated with IBD.

\section{Methods}

Mice. Vim KO mice, obtained from Dr. Albee Messing at the University of Wisconsin, were originally provided by Professor Charles Babinet of the Institute Pasteur $^{21}$. Control mice were generated by back-breeding the KO mice with the WT strain $(129 / \mathrm{SVEV})$ from which they were derived for at least ten generations. Mice were housed in specific pathogen-free conditions at the Animal Maintenance Facility of the University of Michigan Medical Center and used for experiments at 1013 weeks of age. The University of Michigan Committee on Use and Care of Animals reviewed and approved all animal protocols.

In vivo septicemia model. Mice were injected intraperitoneally (IP) with $2 \times 10^{10} \mathrm{cfu}$ of E. coli J96 (American Type Tissue Collection, Manassas, VA). Blood samples were collected 3 and 9 hours post-injection and incubated overnight in tryptic soy broth. Serial dilutions of these cultures were grown for $24-48$ hours on tryptic soy agar plates. At the end of the incubation, cfu were enumerated. Mouse survival was monitored and recorded. 
Collection of peritoneal macrophages. Resident peritoneal macrophages were collected from mice. Peritoneal cells were harvested 4-5 days after injection via peritoneal lavage. Cells were used in experiments either immediately after harvesting or following 4-5 days of culture aimed to enhance macrophage purity. To this end, cells were allowed to adhere to non-tissue-culture-treated plates and cultured in RPMI 1640 supplemented with 10\% heat-inactivated FBS and 5\% penicillin/ streptomycin (Bio-Whittaker, Walkersville, MD) ${ }^{28}$.

Generation of bone marrow-derived macrophages (BMM). BMM were generated according to the method reported by Celada et al. ${ }^{29}$ with certain modifications. Bone marrow cells were harvested from mouse femurs and cultured in RPMI supplemented with $20 \%$ heat-inactivated FBS and 30\% L-cell (a mouse fibroblast cell line that secrets $\mathrm{M}$-CSF) supernatant in non-tissue culture $100 \mathrm{~mm}$ Petri dishes at a concentration of $3-4 \times 10^{6}$ cells per $20 \mathrm{ml}$ media. The cells were incubated at $37^{\circ} \mathrm{C}$ in a humidified $5 \%$ $\mathrm{CO}_{2}$ atmosphere for 5-7 days. Macrophages were harvested in cold PBS prior to use in experiments.

Bacterial phagocytosis and killing assays. Peritoneal macrophages were harvested from individual mice and analyzed separately. Cells were plated in two 24 -well $(1 \times$ 106/well) non-tissue-culture-treated plates in RPMI 1640 supplemented with $10 \%$ FBS and immediately incubated with $5 \times 10^{6}$ colony forming units (cfu) of E. coli $\mathrm{J} 96$. After $45 \mathrm{~min}$ of incubation at $37^{\circ} \mathrm{C}$, the wells were extensively washed to remove nonphagocytosed bacteria. Cells in one of the plates were then lysed using sterile $0.5 \%$ Triton X-100 to analyze bacterial phagocytosis. The second plate was incubated in fresh media for an additional $45 \mathrm{~min}$. Then, cells were lysed to analyze bacterial killing. Serially diluted cell lysates were plated on agar (Difco, Detroit, MI) and incubated overnight at $37^{\circ} \mathrm{C}$. The number of colonies per plate was enumerated. The rate of bacterial killing was calculated using the following formula: [1-(cfu at $90 \mathrm{~min} /$ cfu at $45 \mathrm{~min})] \times 100^{30}$.

Nitric oxide assay. Peritoneal macrophages were harvested from individual mice and analyzed separately. Cells were cultured in 48-well non-tissue-culture treated plates. After 3-4 days, cells were treated with $1 \mu \mathrm{g} / \mathrm{ml}$ lipopolysaccharide (LPS; Sigma, St. Louis, MO) and $50 \mathrm{ng} / \mathrm{ml}$ mouse IFN- $\gamma$ (R\&D Systems, Minneapolis, MN) for 48 hours. Supernatants were collected and analyzed for nitric oxide concentration by measuring the levels of $\mathrm{NO}_{2}{ }^{-}$, a breakdown product of nitric oxide, using the Griess Reagent System (Promega, Madison, WI). Dye intensity was measured within $30 \mathrm{~min}$ using a plate reader equipped with a $450 \mathrm{~nm}$ filter. Results were calculated using a nitrite standard curve.

Oxidative burst assay. Peritoneal macrophages were harvested from individual mice and analyzed separately. Cells were washed, suspended in DPBS supplemented with $10 \mathrm{mM}$ glucose, and transferred to a 96 -well plate or $12 \mathrm{~mm} \times 50 \mathrm{~mm}$ polypropylene tubes (Turner Designs, Sunnyvale, CA) at a concentration of $1.5 \times 10^{6}$ cells per $50 \mu \mathrm{l}$ of media. The overall generation of ROS metabolites $\left(\mathrm{H}_{2} \mathrm{O}_{2}\right.$ and $\left.\mathrm{O}_{2}{ }^{-}\right)$was measured by detecting chemiluminescence of phagocytes treated with $10 \mathrm{ng} / \mathrm{ml}$ PMA in the presence of $50 \mu \mathrm{M}$ luminal (Sigma, St. Louis, MO) for $5 \mathrm{~min}$ at $37^{\circ} \mathrm{C}^{31}$. Light emission was measured using a TD-20/20 Luminometer (Turner Designs, Sunnyvale, CA) or by the Tecan GENios plate reader (Phenix, Australia)

$\mathrm{H}_{2} \mathrm{O}_{2}$ detection. BMM were plated in 96-well plates. Secretion of $\mathrm{H}_{2} \mathrm{O}_{2}$ was determined colorimetrically using Amplex Red reagent (Molecular Probes, Eugene, OR) according to the instructions of the manufacturer and as previously described ${ }^{32}$. Briefly, a solution containing $50 \mu \mathrm{M}$ Amplex Red reagent and $10 \mathrm{U} / \mathrm{ml} \mathrm{HRP}$ was prepared in PBS, and $0.1 \mathrm{ml}$ of the suspension was added to the BMM cultures $(5 \times$ $10^{5}$ per well). Cells were incubated at $37^{\circ} \mathrm{C}$ for $60 \mathrm{~min} . \mathrm{H}_{2} \mathrm{O}_{2}$ concentrations in the culture media were determined using a standard curve generated with known $\mathrm{H}_{2} \mathrm{O}_{2}$ concentrations with a detection limit of $0.625 \mathrm{nM}$. Samples were measured at A560 nM wavelength using a Tecan GENios plate reader (Phenix, Australia).

NADPH detection. NADPH concentrations were determined by NADP/NADPH fluorimetric detection kit (Cell Technology Inc, Mountain View, CA). Briefly, $1 \times$ $10^{6} \mathrm{BMM}$ were harvested and spun down. The pellets were analyzed according to the manufacturer's instructions. NADPH concentrations ranging between $3.9 \mathrm{nM}-$ $250 \mathrm{nM}$ were determined using a standard curve generated with known concentrations of NADPH.

Knocking down vimentin expression with a short hairpin RNA (shRNA). A pLKO.1 vector expressing an shRNA targeting vimentin and an empty control vector were purchased from Open Biosystems (Thermo Scientific, Huntsville, AL). Lentiviral particles were generated in HEK293T cells using the packaging plasmids psPAX2 and pMD2.G as described in detail at http://www.broadinstitute.org/rnai/ public/resources/protocols. THP-1 (a human monocytic cell line) cells were infected with the lentiviral particles. Stably-transduced cells were selected by puromycin resistance. Expression of vimentin in tranduced and control cells was assessed via immunoblotting.

High-performance liquid chromatography (HPLC) analysis of intracellular GSH and GSSG. Cultured BMM were infected with E. coli for $45 \mathrm{~min}$, stimulated with LPS $(100 \mathrm{ng} / \mathrm{ml})$ overnight, or left untreated. For analysis of intracellular GSH and GSSG, cells were washed three times with ice-cold PBS, resuspended in PBS, and then detached by gentle scraping on ice. Aliquots of the cell suspensions were mixed with equal volumes of metaphosphoric acid solution $\left(16.8 \mathrm{mg} / \mathrm{ml} \mathrm{HPO}_{3}, 2 \mathrm{mg} / \mathrm{ml} \mathrm{EDTA}\right.$, and $9 \mathrm{mg} / \mathrm{ml} \mathrm{NaCl}$ ). Protein-free cell lysates were either stored at $-80^{\circ} \mathrm{C}$ or

derivatized immediately with monoiodoacetic acid at a final concentration of $7 \mathrm{mM}$, followed by 2 , 4-dinitrofluorobenzene $(1.5 \% \mathrm{v} / \mathrm{v}$ in absolute ethanol) and analyzed by HPLC as previously described ${ }^{33,34}$. To measure protein concentrations, aliquots of the cell suspensions were mixed with equal volumes of lysis buffer $(0.1 \mathrm{M}$ sodium phosphate, $\mathrm{pH} 7.4$, containing $0.1 \%$ Triton-X $100,10 \mu \mathrm{l} / \mathrm{ml}$ protease inhibitor cocktail (Sigma), $25 \mu \mathrm{g} / \mathrm{ml}$ tosyllysine chloromethylketone, and $5 \mu \mathrm{g} / \mathrm{ml}$ phenylmethylsulfonyl fluoride (Sigma, St. Louis, MO)) and centrifuged at $12,000 \times g$ for $10 \mathrm{~min}$ at $4^{\circ} \mathrm{C}$. Protein concentrations in the cell lysates were measured by the Bradford method (Bio-Rad) using bovine serum albumin as a standard ${ }^{33}$.

Western blotting. THP-1 cells were lysed in 2\% SDS and samples were separated by $10 \%$ SDS-polyacrylamide gel electrophoresis (PAGE). The gel was transferred to a PVDF membrane and probed with monoclonal anti-vimentin antibodies (Santa Cruz Biotechnology, Santa Cruz, CA) followed by an appropriate horseradish peroxidaseconjugated secondary antibody. Chemiluminescent signal was detected using the Super Signal West Pico system (Pierce, Rockford, IL).

Cell fractionation, immunoprecipitation, and Western blotting. Peritoneal macrophages $\left(5 \times 10^{6}\right)$ or BMM $\left(2 \times 10^{7}\right)$ were stimulated for 5 min with $1 \mathrm{ng} / \mathrm{ml}$ PMA. Treated and untreated cells were lysed and sonicated in ice-cold lysis buffer containing $150 \mathrm{mM}$ Tris-HCL ( $\mathrm{pH} 8.0$ ), $100 \mathrm{mM} \mathrm{NaCl}$, and protease inhibitors (Roche Diagnostics, Indianapolis, IN). Cell lysates underwent ultracentrifugation at $100,000 \mathrm{~g}$ for $90 \mathrm{~min}$ at $4{ }^{\circ} \mathrm{C}$. The cytosolic (soluble) fraction was harvested. The membrane (insoluble) fraction was washed, resuspended and sonicated in lysis buffer. The membrane and cytosolic fractions were subjected to immunoblotting or to coimmunoprecipitation. To this end, samples were incubated overnight at $4{ }^{\circ} \mathrm{C}$ with polyclonal anti-vimentin antibodies $(1: 50$; raised by Harlen Bioproducts for Science, INC. (Madison, WI) and purified over a His-vimentin column as previously described ${ }^{35}$ ) or control rabbit IgG antibodies. Protein A and G- Sepharose were added to each sample. Then, samples were incubated for 2 hours at $4^{\circ} \mathrm{C}$. Beads were washed 5 times with $300 \mathrm{mM} \mathrm{NaCl}$ in PBS, and samples were separated by $10 \%$ SDSpolyacrylamide gel electrophoresis (PAGE). The gel was transferred to PVDF membrane and probed with monoclonal anti-p47phox antibodies (Santa Cruz Biotechnology, Santa Cruz, CA) followed by an appropriate horseradish peroxidaseconjugated secondary antibody. Chemiluminescent signal was detected using the Super Signal West Pico system (Pierce, Rockford, IL).

Immunohistochemistry. Cells were cultured in a glass chamber slide system (Nalge Nunc International, Naperville, IL). Cultures were washed with PBS and fixed in $4 \%$ paraformaldehyde for $10 \mathrm{~min}$ at $4{ }^{\circ} \mathrm{C}$. Cells were washed again, blocked with $0.2 \%$ BSA in PBS, and then washed with $0.1 \%$ saponin (Sigma, St Louis, MO) in PBS. Cells were incubated with rabbit anti-vimentin (raised by Harlen Bioproducts for Science, INC) and mouse anti-p47phox (Santa Cruz Biotechnology, Santa Cruz, CA) antibodies. Slides were washed and blocked with goat sera prior to incubation with Alexa 488 (fluorescein) goat-anti mouse or Alexa 594 (rhodamine) goat anti-rabbit antibodies (Molecular Probes, Eugene, OR). Slides were washed and mounted with Prolong Gold reagent (Molecular Probes, Eugene, OR). Confocal fluorescence microscopy analysis was performed using a 60X lens of a Zeiss LSM 510 laser scanning microscope.

DSS-induced acute colitis. Acute colitis in mice was induced by administration of $4.5 \%(\mathrm{w} / \mathrm{v})$ DSS (molecular mass $36-50 \mathrm{kDa}$; MP Biologicals, Solon, $\mathrm{OH}$ ) in the drinking water that was provided ad libitum for 8 consecutive days (the solutions were made fresh every 3 days), followed by 6 days of normal drinking water. Body weight and the presence of blood in the stool were monitored every other day from days 1 through 14. The latter was determined using the Hemoccult test (Beckman Coulter, Fullerton, CA) and visual inspection. Stool blood scores were assigned as follows: 0, no blood; 1 , positive hemoccult; 2 , visible traces of blood; 3 , gross rectal bleeding ${ }^{36}$.

Histopathology and Immunohistochemistry. Six days after the end of the DSS treatment (day 14), mice were sacrificed and the entire colon was excised, washed, fixed in $10 \%$ buffered formaldehyde, and embedded in paraffin. Tissue sections were stained with hematoxylin \& eosin (H\&E) and scored by a pathologist in a blinded fashion according to the colitis index histological scoring system used by Law et $\mathrm{al}^{36,37}$ Briefly, sections were evaluated for the presence of inflammation as manifested by immune cell infiltration, damaged crypts, submucosal edema, submucosal hemorrhage, and transmural involvement. The value of the colitis index score increases with the severity of the inflammation; the score ranges from 0 to 40 . Animals who died were given the highest possible score of 40 .

Bacterial culture. Total mesenteric lymph node tissue was cultured in LB media at $37^{\circ} \mathrm{C}$ under aerobic conditions. After 24 hours, cultures were transferred to LB agar plates at different dilutions. Colony-forming units (CFU) per $\mathrm{ml}$ of initial culture volume were determined and normalized to tissue weight.

Statistical Analysis. Data are presented as means \pm SEM and are representative of at least two independent experiments. Data were evaluated by unpaired Student's $t$-test. Survival curves were compared using the log-rank test. Statistical analysis was 
performed using GraphPad Prism (GraphPad Software, La Jolla, CA). P values less than 0.05 were considered statistically significant.

1. Evans, R. M. Vimentin: the conundrum of the intermediate filament gene family. Bioessays 20, 79-86 (1998).

2. Traub, P. Intermediate Filaments A Review. (Springer-Verlag, 1985).

3. Fuchs, E. \& Weber, K. Intermediate filaments: structure, dynamics, function, and disease. Annu Rev Biochem 63, 345-382 (1994).

4. Eckes, B. et al. Impaired wound healing in embryonic and adult mice lacking vimentin. J Cell Sci 113 ( Pt 13), 2455-2462 (2000).

5. Eckes, B. et al. Impaired mechanical stability, migration and contractile capacity in vimentin-deficient fibroblasts. J Cell Sci 111 ( Pt 13), 1897-1907 (1998).

6. Nieminen, M. et al. Vimentin function in lymphocyte adhesion and transcellular migration. Nat Cell Biol 8, 156-162 (2006).

7. Cifuentes, M. E. \& Pagano, P. J. Targeting reactive oxygen species in hypertension. Curr Opin Nephrol Hypertens 15, 179-186 (2006)

8. Touyz, R. M., Yao, G., Quinn, M. T., Pagano, P. J. \& Schiffrin, E. L. p47phox associates with the cytoskeleton through cortactin in human vascular smooth muscle cells: role in NAD(P)H oxidase regulation by angiotensin II. Arterioscler Thromb Vasc Biol 25, 512-518 (2005).

9. Sumimoto, H. Structure, regulation and evolution of Nox-family NADPH oxidases that produce reactive oxygen species. FEBS J 275, 3249-3277 (2008).

10. Mor-Vaknin, N., Punturieri, A., Sitwala, K. \& Markovitz, D. M. Vimentin is secreted by activated macrophages. Nat Cell Biol 5, 59-63 (2003).

11. Weyand, C., Goronzy, J., Fathman, C. G. \& O'Hanley, P. Administration in vivo of recombinant interleukin 2 protects mice against septic death. J Clin Invest 79, 1756-1763 (1987).

12. MacMicking, J., Xie, Q. W. \& Nathan, C. Nitric oxide and macrophage function. Annu Rev Immunol 15, 323-350 (1997).

13. Krieglstein, C. F. et al. Regulation of murine intestinal inflammation by reactive metabolites of oxygen and nitrogen: divergent roles of superoxide and nitric oxide. J Exp Med 194, 1207-1218 (2001).

14. Tolstonog, G. V., Shoeman, R. L., Traub, U. \& Traub, P. Role of the intermediate filament protein vimentin in delaying senescence and in the spontaneous immortalization of mouse embryo fibroblasts. DNA Cell Biol 20, 509-529 (2001).

15. Zhang, J. et al. Increased contribution of L-arginine-nitric oxide pathway in aorta of mice lacking the gene for vimentin. J Cardiovasc Pharmacol 38, 552-560 (2001).

16. Allen, L. A. et al. Transient association of the nicotinamide adenine dinucleotide phosphate oxidase subunits p47phox and p67phox with phagosomes in neutrophils from patients with X-linked chronic granulomatous disease. Blood 93, 3521-3530 (1999).

17. Boxer, L. Disorders of phagocyte function. 18th edn, (Saunders Elsevier, 2007).

18. Smith, A. M. et al. Disordered macrophage cytokine secretion underlies impaired acute inflammation and bacterial clearance in Crohn's disease. J Exp Med 206, 1883-1897 (2009).

19. Oz, H. S., Chen, T. S., McClain, C. J. \& de Villiers, W. J. Antioxidants as novel therapy in a murine model of colitis. J Nutr Biochem 16, 297-304 (2005).

20. Wirtz, S., Neufert, C., Weigmann, B. \& Neurath, M. F. Chemically induced mouse models of intestinal inflammation. Nat Protoc 2, 541-546 (2007).

21. Colucci-Guyon, E. et al. Mice lacking vimentin develop and reproduce without an obvious phenotype. Cell 79, 679-694 (1994).

22. Galou, M. et al. Disrupted glial fibrillary acidic protein network in astrocytes from vimentin knockout mice. J Cell Biol 133, 853-863 (1996).

23. Terzi, F. et al. Reduction of renal mass is lethal in mice lacking vimentin. Role of endothelin-nitric oxide imbalance. J Clin Invest 100, 1520-1528 (1997).

24. Zou, Y., He, L. \& Huang, S. H. Identification of a surface protein on human brain microvascular endothelial cells as vimentin interacting with Escherichia coli invasion protein IbeA. Biochem Biophys Res Commun 351 (2006).

25. Chi, F. et al. Vimentin-mediated signalling is required for IbeA+ E. coli K1 invasion of human brain microvascular endothelial cells. Biochem J 427, 79-90 (2010).

26. Tolstonog, G. V., Mothes, E., Shoeman, R. L. \& Traub, P. Isolation of SDS-stable complexes of the intermediate filament protein vimentin with repetitive, mobile, nuclear matrix attachment region, and mitochondrial DNA sequence elements from cultured mouse and human fibroblasts. DNA Cell Biol 20, 531-554 (2001).

27. Fritz, T., Niederreiter, L., Adolph, T., Blumberg, R. S. \& Kaser, A. Crohn's disease: NOD2, autophagy and ER stress converge. Gut 60, 1580-1588 (2011)

28. Cohn, Z. A. \& Benson, B. The Differentiation of Mononuclear Phagocytes. Morphology, Cytochemistry, and Biochemistry. J Exp Med 121, 153-170 (1965).
29. Celada, A., Gray, P. W., Rinderknecht, E. \& Schreiber, R. D. Evidence for a gamma-interferon receptor that regulates macrophage tumoricidal activity. J Exp Med 160, 55-74 (1984).

30. Matsukawa, A. et al. Pivotal role of the CC chemokine, macrophage-derived chemokine, in the innate immune response. J Immunol 164, 5362-5368 (2000).

31. Albrecht, D. \& Jungi, T. W. Luminol-enhanced chemiluminescence induced in peripheral blood-derived human phagocytes: obligatory requirement of myeloperoxidase exocytosis by monocytes. J Leukoc Biol 54, 300-306 (1993)

32. Serezani, C. H., Aronoff, D. M., Jancar, S., Mancuso, P. \& Peters-Golden, M. Leukotrienes enhance the bactericidal activity of alveolar macrophages against Klebsiella pneumoniae through the activation of NADPH oxidase. Blood 106, 1067-1075 (2005).

33. Mosharov, E., Cranford, M. R. \& Banerjee, R. The quantitatively important relationship between homocysteine metabolism and glutathione synthesis by the transsulfuration pathway and its regulation by redox changes. Biochemistry 39, 13005-13011 (2000).

34. Garg, S. K., Banerjee, R. \& Kipnis, J. Neuroprotective immunity: T cell-derived glutamate endows astrocytes with a neuroprotective phenotype. J Immunol 180, 3866-3873 (2008).

35. Kappes, F., Scholten, I., Richter, N., Gruss, C. \& Waldmann, T. Functional domains of the ubiquitous chromatin protein DEK. Mol Cell Biol 24, 6000-6010 (2004).

36. Berndt, B. E., Zhang, M., Chen, G. H., Huffnagle, G. B. \& Kao, J. Y. The role of dendritic cells in the development of acute dextran sulfate sodium colitis. J Immunol 179, 6255-6262 (2007).

37. Law, D. J., Labut, E. M., Adams, R. D. \& Merchant, J. L. An isoform of ZBP-89 predisposes the colon to colitis. Nucleic Acids Res 34, 1342-1350 (2006).

\section{Acknowledgments}

We thank Mike Khodadoust for technical advice and intellectual support, and Donna Gschwend, Anjan Kumar Saha, Jen Lewis, and Ferdinand Kappes for scientific input and manuscript preparation. Special thanks for Dr. Barbara S. Adams for editing the manuscript and Bruce Donohoe (University of Michigan Microscopy \& Imaging Core) for his help in acquiring the confocal microscopy images. This work was supported by grants to D.M.M. from the Arthritis Foundation and the Burroughs Welcome Fund. N.M.-V. was supported by a grant from the Arthritis Foundation, NIH grant T32CA88784-03 through the University of Michigan Tumor Immunology Training Program, the Rheumatic Disease Core Center of the University of Michigan (5 P30 AR048310-07), the Post-Doctoral Translational Scholars Program (UL1RR024986), the Gastrointestinal Peptide Research Center (5 P30 DK34933), and R03 AR056748-01 and K01 AR055620 from the NIH. R.B. and S.K.G. were supported by NIH grant DK64959. A.P. was supported by Merit Review funding and a Research Enhancement Award Program (REAP) grant from the Department of Veterans Affairs. M.P.-G. was supported by NIH grant HL058897 and C.H.C.S. was supported by an American Lung Association Senior Postdoctoral Research Fellowship and grants K991R00 and HL103777 from the NIH. M.J.G.-H. was supported by a Rackham Merit Fellowship, the Mechanisms of Microbial Pathogenesis Training Grant from the University of Michigan, and by an NIH Ruth L. Kirschstein NRSA Individual Predoctoral Fellowship to Promote Diversity in Health-Related Research grant 1F31CA150523-01. J.Y.K. was supported by DK087708-01 from the NIH.

\section{Author contributions}

M.L., Y.Y., S.K.G., A.J., M.D.S. preformed the experiments and analyzed the data. M.G. and S.T.T. preformed experiments, analyzed the data and provided advice in writing the manuscript. C.H.C., A.P., N.C.E., R.B., M.P.G., J.Y.K. provided advice in regarding experiments design and conclusions. D.M.M. provided advice regarding experiment design and manuscript writing. N.M.V. design, preforms, analyzed and wrote the paper with much contribution of all the authors above.

\section{Additional information}

Competing financial interests: The authors declare no competing financial interests.

License: This work is licensed under a Creative Commons

Attribution-NonCommercial-NoDerivs 3.0 Unported License. To view a copy of this license, visit http://creativecommons.org/licenses/by-nc-nd/3.0/

How to cite this article: Mor-Vaknin, N. et al. Murine Colitis is Mediated by Vimentin. Sci. Rep. 3, 1045; DOI:10.1038/srep01045 (2013). 